\title{
The Relationship Between Rheumatoid Arthritis and COVID-19
}

\author{
Ozan Emre EYUPOGLU* and Irem ATES \\ Department of Biochemistry, School of Pharmacy, Istanbul Medipol University, Turkey \\ *Corresponding author: Ozan Emre EYUPOGLU, Department of Biochemistry, School of Pharmacy, Istanbul Medipol \\ University, Turkey
}

\section{ARTICLE INFO}

Received: 慧 August 31, 2021

Published: 慧 September 07, 2021

Citation: Ozan Emre EYUPOGLU, Irem ATES. The Relationship Between Rheumatoid Arthritis and COVID-19. Biomed J Sci \& Tech Res 38(4)-2021. BJSTR. MS.ID.006177.

Keywords: Rheumatoid Arthritis; Synovitis; COVID-19; Outbreak

\section{ABSTRACT}

Rheumatoid arthritis is an autoimmune disorder that affects $0.5 \%$ to $1 \%$ of the general population. It is more frequent in women because of hormonal reasons. Although the etiopathology is not known exactly, the general symptoms caused by synovial inflammation are characterized by pain and swelling. Rheumatoid disease is considered to be an incurable disease but can stay in remission with a wide range of pharmacological spectrum from analgesics to biological agents. The outbreak caused by the new type of coronavirus infection in 2019 negatively affected the whole world. People with weakened immune systems, elderly and autoimmune diseases suffered from COVID-19 disease or resulted in their death. It has a high risk of transmission and a low mortality rate. COVID-19 disease may show itself in severe clinical pictures such as organ failure or cytokine storm. It is extremely challenging for a patient to survive such a clinical picture and requires serious treatment.

Abbreviations: RA: Rheumatoid Arthritis; ESR: Erythrocyte Sedimentation Rate; CRP: C-Reactive Protein; Ig G: Immunoglobulin G; RA: Rheumatoid Arthritis; RF: Rheumatoid Factor; ACPA: Antibodies to Citrullinated Peptides/Proteins; CCP: Cyclic Citrullinated Peptides; ELISA: Enzyme-Linked Immunosorbent Assay; MTX: Methotrexate; N: Nucleocapsid Protein; S: Spike Glycoprotein; E: Envelope Glycoprotein; M: Membrane Protein; CTD: C-Terminal Peptidase; TMPRSS2: Type 2 Transmembrane Serine Protease; RBD: Receptor-Binding Domain; NAAT: Nucleic Acid Amplification Test; WBC: White Blood Cell; LDH: Lactate Dehydrogenase; AST: Aspartate Aminotransferase; ALT: Alanine Aminotransferase; CK: Creatine Kinase; CT: Computed Tomography; HRCT: High-Resolution CT

\section{Introduction}

Rheumatoid Arthritis (RA) is an autoimmune systemic multidisciplinary disease that affects $1 \%$ of the general population [1]. The aetiology of this disease, which results in joint destruction, is characterized by chronic inflammation, progresses cumulatively, negatively affects many systems of the body, and is three times more common in women than in men [2]. Some factors such as genetic, environmental, age and gender are crucial in its progression [3]. The disease can be diagnosed by increasing inflammatory biomarkers in the inflamed synovium and distinguished from other types of arthritis [4]. Together with the earth-shaking COVID-19 outbreak that started in Wuhan, China in December 2019, some biomarkers in RA have become crucial [4,5]. The most popular markers (IL-6) that shed light on the progression and pathology of the disease are markers such as Interleukin-6, ACPA, and anti-CCP. In rheumatoid arthritis disease, inflammation shows a substantial increase [4].

\section{Aetiology and Pathology of Rheumatoid Arthritis}

Although the aetiology of RA is not known exactly, it occurs in different clinical pictures that are considered to be involved in infection, genetic, environmental, and endocrine dysregulation [3]. Inflammation of the synovium is a pathological event that destroys articular cartilage and bone. The most crucial condition 
that distinguishes it from other articular pathologies is joint destruction [6]. Genetic factors explicitly play a role in the severity and progression of rheumatoid arthritis. The prevalence of RA, which is $1 \%$ in the general population, is $12-15 \%$ in monozygotic (identical) twins, while this rate is approximately 2-5\% in dizygotic twins or first-degree relatives [7]. Environmental factors in addition to genetic factors ( $50 \%$ ) have a role in the pathogenesis of RA. One of the most important environmental factors is infections. Many pathogens have been argued in the pathogenesis of RA. Although Mycobacterium tuberculosis, Proteus mirabilis, Escherichia coli, Epstein-Barr virus, Parvovirus 19, and some retroviruses have been blamed, no organism could not be held liable [8].

Rheumatoid Arthritis is a disease that is three times more common in women than in men. Although the basis of the gender difference cannot be set out in full, the higher incidence of RA in women can be based on hormones [2]. To penetrate the joint space, they must pass through the layer of synovial tissue, which is very thin in healthy joints, but consists of a thick lining layer of macrophages and fibroblastoid synovial cells in rheumatoid joints. Deeper parts of synovial tissue accumulate lymphoid cells histologically organized together with dendritic cells such as lymphatic nodules. Deeper areas of the synovial tissue, in parallel with dendritic cells such as nodules, stromal fibroblasts transform from a long-stretched appearance to small round cells, increase in number and are present with a proliferative response. These pathologies refer to chronicity and severity. These pathologies are not homogeneous within the same joint. B and T cells are not only found much more in the stromal area, but they are also usually found around blood vessels and are accompanied by dendritic cells [9].

\section{Clinical Characteristics of Rheumatoid Arthritis}

Rheumatoid arthritis is a severe disease that may have many extra-articular manifestations. Extra-articular involvement may occur in $40 \%$ of patients at the onset and course of the disease. Extra-articular involvement is associated with increased mortality in RA. The life expectancy of patients with RA without extraarticular manifestations is like that of the general population [10]. Pulmonary involvement is frequent in patients with rheumatoid arthritis. Mortality rates are high as a result of pulmonary involvement. Although asymptomatic, the prognosis of Pleural disease is frequent in rheumatoid arthritis patients [11]. Rheumatoid nodules in the lung occur mostly in seropositive male patients with synovitis. It rarely occurs to diagnose in the clinic. It can be seen alone or in the form of a bunch of grapes in radiographic imaging [10]. Interstitial lung disease is the most crucial pulmonary involvement in rheumatoid arthritis [12]. The prevalence of the involvement of the cardiovascular system is high in rheumatoid arthritis. Cardiovascular disease, which usually occurs in patients with early RA, shortens the patient's life by 5 to 15 years and is characterized by a mortality rate of $42 \%$.

Pericarditis is the most common heart condition. Many patients with early RA may have pericarditis or develop pericarditis before RA. Although less than $15 \%$ of RA patients had clinical symptoms, the ECG showed that $20 \%$ to $50 \%$ of patients had pericardial involvement emerging as chest pain or shortness of breath. Heart failure is the most important cause of death among RA patients. Arrhythmia is another common cardiac complication in RA patients that may occur secondary to conduction abnormalities due to local ischemia, rheumatoid nodules, amyloidosis, or heart failure. The main cause of cardiovascular disease contributing to cardiac dysfunction remains unclear. Therefore, early diagnosis and prevention of cardiac dysfunction are crucial [13]. Anaemia is one of the most common extra-articular symptoms of RA. The cause of Anaemia in RA is multifactorial. These are drug-induced malnutrition, gastrointestinal bleeding, bone marrow suppression, and ineffective erythropoiesis. Thrombocytosis is a common finding in active RA and is associated with the number of actively inflamed joints. Lymphadenopathy is sometimes observed in active RA, usually seen as benign follicular hyperplasia on biopsy [10].

\section{Laboratory Findings in Rheumatoid Arthritis}

The first laboratory tests should include complete blood count, biochemistry panel, erythrocyte sedimentation rate (ESR) and/or C-reactive protein (CRP), complete urinalysis, rheumatoid factor titer, and / or anti-CCP antibodies in patients examined with a preliminary diagnosis of RA [4]. Anaemia, in which hematocrit levels change, can be found in a minority of patients with RA. In most cases, decreased erythrocyte (red cell) mass is the outcome of Anaemia of chronic disease. The cause of Anaemia is usually not an iron deficiency, but an inadequate response to erythropoietin due to inflammation [14]. Although ESR and CRP levels are significant for differentiating non-inflammatory diseases such as osteoarthritis or fibromyalgia from RA, they do not show specificity for diagnosing RA. It might be used to evaluate RA disease activity [15]. CRP levels fluctuate faster than ESR during illness. Anaemia can change the ESR value. It is very useful to check CRP in addition to ESR. CRP synthesized by the liver has pro-inflammatory effects such as activating the classical complement pathway and reducing the IL-6 receptor concentration.

CRP has a very short half-life, and its concentration rises within a few hours with inflammation and peaks in 2 to 3 days. After the inflammation subsides, its concentration quickly returns to normal. In healthy people, the CRP level is below $0.2 \mathrm{mg} / \mathrm{dL}$, but this level may reach up to $1 \mathrm{mg} / \mathrm{dL}$ depending on microtraumas. It can also be used to determine prognosis and monitor disease severity in RA [16]. $\alpha 1$-acid glycoprotein analysis and secretion increase with 
the stimulation of cytokines such as IL-1 and TNF- $\alpha$. CRP and $\alpha 1-$ acid glycoprotein were found to be most useful in assessing RA disease activity. High levels of CRP are associated with mortality and the need for intubation. CRP can increase due to many reasons $[16,17]$. A factor in the serum of patients with rheumatoid arthritis (RA) causing the agglutination of red blood cells sensitized with immunoglobulin G (Ig G) antibodies, was first described in 1940. Later, this factor was named the rheumatoid factor (RF). Rheumatoid factors (RFs) are antibodies directed to the Fc region (crystallizable) of human IgG molecules. These are the hallmark autoantibodies of rheumatoid arthritis (RA), an extravascular immune complex disease [18].

The most commonly used test serum in the diagnostic criteria of rheumatoid arthritis is the measure of rheumatoid factor (RF). Its sensitivity is $60 \%$ to $90 \%$, and its specificity is as high as $85 \%$ [19]. The presence or absence of antibodies to citrullinated peptides/ proteins (ACPA) is an important parameter that helps a clinician set a diagnosis of early rheumatoid arthritis and hence, initiate treatment. Anti-cyclic citrullinated peptides (anti-CCP) are a type of IgG autoantibodies recognizing citrullinated peptides and offering improved specificity in the early diagnosis of RA compared to RF. Traditionally Anti-CCP testing has been performed using ELISA assays [20]. Administration of citrulline-containing peptide (CCP) in the enzyme-linked immunosorbent assay (ELISA) revealed the presence of anti-CCP autoantibodies in $76 \%$ of rheumatoid arthritis sera with $95 \%$ to $100 \%$ specificity. When this high specificity is combined with the presence of the disease, even before the early onset of the disease, it suggests a role for these autoantibodies in the pathogenesis of rheumatoid arthritis [21].

\section{Treatment of Rheumatoid Arthritis}

Non-steroidal anti-inflammatory drugs, glucocorticoids, disease-modifying drugs, and biological agents are used to treat rheumatoid arthritis. Methotrexate (MTX) is the first drug of choice in patients with aggressive RA, and it is an immunosuppressive drug that can make DNA synthesis irregular by inhibiting folic acid (vitamin B9) metabolism [22]. Based on in vivo and ex vivo tests, it increases adenosine and anti-inflammatory cytokine levels, while decreasing proinflammatory cytokines such as IL-6 and IL-1 $\beta$. The exacerbation reason of the disease after the discontinuation of drug use is also related to these features [23]. Leflunomide, which was approved in 1998 and started to be used in active RA disease, is a prodrug. It turns into its active metabolite in the liver, stops the growth of lymphocytes, and inhibits $\mathrm{T}$ cell proliferation as pyrimidine synthesis inhibition [24].

\section{Aetiology and Pathology of SARS-CoV-2}

SARS-CoV-2 is surrounded by an envelope containing the viral nucleocapsid. It is positive polarity, single-stranded RNA virus with virion diameters ranging from 60 to $140 \mathrm{~nm}$ and distinctive spikes of 9 to $12 \mathrm{~nm}$. This virus is divided into four major structural proteins, namely, nucleocapsid protein $(\mathrm{N})$, spike glycoprotein $(\mathrm{S})$, envelope glycoprotein (E), and membrane protein (M) [25]. Spike glycoprotein $(\mathrm{S})$ mediates attachment of the virus to the host cell surface receptors and subsequent fusion between the viral and host cell membranes to facilitate viral entry into the host cell [26]. The envelope glycoprotein (E) is the smallest of the major structural proteins (between $8 \mathrm{kDa}$ and $12 \mathrm{kDa}$ ) and has the lowest copy number in the lipid envelope of mature virus particles [27]. The nucleocapsid protein $(\mathrm{N})$ is the most abundant protein in virusinfected cells. The $\mathrm{N}$ protein, which ranges from $43 \mathrm{kDa}$ to $50 \mathrm{kDa}$, is the protein component of the helical nucleocapsid and is thought to bind the genomic RNA in a beads-on-a-string fashion. Membrane protein (M) is the most abundant component of coronaviruses and defines the shape of the viral envelope. The pre-glycosylated M polypeptide ranges in size from $25 \mathrm{kDa}$ to $30 \mathrm{kDa}$ (221-262 amino acids) [28].

SARS-CoV-2 infects the lower respiratory tract, causing pneumonia. Causes of death are due to resulting in respiratory dysfunction and hyper inflammation [29]. ACE-2 is the S proteinmediated cell entry receptor for coronavirus and provides a direct binding site for S proteins [30]. The ACE-2 protein contains the $\mathrm{N}$-terminal peptidase domain and the C-terminal peptidase (CTD) domain. The S1 protein detected in SARS-CoV-2 provides the attachment of cellular membranes. The S2 protein performs membrane fusion [22]. The entry of the virus into the cell occurs via endocytosis as a result of membrane fusion [31]. Type 2 transmembrane serine protease (TMPRSS2) cleaves the ACE-2 receptor, activates the $\mathrm{S}$ protein, and mediates its entry into the cell [30]. To understand the infection potential of SARS-CoV-2 in humans, it is necessary to analyze the receptor-binding domain (RBD) of the S protein in contact with ACE-2. In a study, it was shown that the affinity of SARS-CoV-2-CTD is higher than its affinity for SARS-CoV-2-RBD [22].

\section{Clinical Course of COVID-19}

It was found that the COVID-19 outbreak, which started in December 2019, can be transmitted up to distances of 7 to 8 meters through small droplets emitted directly during sneezing [32]. The symptoms of COVID-19 infection appear after an incubation period of approximately 5.2 days [33]. The most common symptoms at the onset of infection are fever, fatigue, dry cough, myalgia, and shortness of breath; fewer common symptoms are headache, dizziness, abdominal pain, diarrhoea, nausea, vomiting, anorexia, and conjunctivitis [22,34]. If we look at the course of the COVID-19 disease on the respiratory system, lung functions deteriorate, and dyspnoea emerges after 5-7 days. If there is a comorbid disease in the patient's history, this period might be shorter. This period may be longer in young patients with good medical history. The subsequent development of hypoxemia emerges with the disruption of gas 
exchange characterized by pulmonary inflammation. When oxygen saturation (Sp02) is $<93$ at the time of the onset of hypoxemia symptoms, the first treatment should be oxygen support [22,35].

Cytokine release syndrome is a life-threatening systemic inflammatory syndrome involving elevated levels of circulating cytokines and immune-cell hyperactivation that can be triggered by various therapies, pathogens, cancers, autoimmune conditions, and monogenic disorders. From a historical perspective, cytokine storm was previously referred to as an influenza-like syndrome that occurred after systemic infections such as sepsis and after immunotherapies such as sepsis. The plague (Yersinia pestis infection), known as Black Death, has led to major pandemics and triggers alveolar macrophages to produce excessive amounts of cytokines, resulting in cytokine storm [36]. Cytokine storm is an umbrella term encompassing several disorders of immune dysregulation characterized by constitutional symptoms, systemic inflammation, and multiorgan dysfunction that can lead to multiorgan failure if inadequately treated. Patients who develop cytokine storm may have high fever, fatigue, anorexia, headache, rash, diarrhoea, myalgia and neuropsychiatric findings. These symptoms may be due directly to cytokine induced tissue damage or acute-phase physiological changes or may result from immune cell-mediated responses [37].

\section{COVID-19 Laboratory, Radiological Findings and Tests}

According to the COVID-19 diagnosis and treatment guidelines, nucleic acid testing is recommended first in clinically suspected cases to confirm the disease. There are two main methods of SARSCoV-2 nucleic acid detection: gene sequencing and nucleic acid amplification test (NAAT) [38]. Haematological biomarkers: White blood cell (WBC) decreases in $80 \%$ of patients and $72.3 \%$ of patients also develop lymphocytopenia. Compared with moderate cases, severe COVID-19 patients have a significantly lower lymphocyte count [39]. The biomarker lactate dehydrogenase (LDH) attracted attention, especially in severe cases. In addition, it is followed by aspartate aminotransferase (AST), alanine aminotransferase (ALT), creatine kinase (CK), and creatinine [40]. LDH, troponin I, and creatinine increase, and hypoalbuminemia may develop in progressive cases causing mortality [41].

Radiological evaluation of suspected COVID-19 patients is required for early detection of thoracic involvement, particularly in emergency units, while waiting for definitive diagnosis of SARS-CoV-2 by real-time reverse transcription-polymerase chain reaction (RT-PCR) which has a gold standard sensitivity of $60 \%$ to $70 \%$ for diagnosis [42-44]. Chest and more specifically pulmonary involvement is the most common site of organ involvement in COVID-19. For Chinese radiologists, the use of computed tomography (CT), especially high-resolution CT (HRCT), along with the patient's symptoms, are the most common approaches to diagnose and stage the severity of pulmonary involvement of COVID-19. The rate of missed COVID-19 diagnosis by CT is $3.9 \%$ [45]. It is crucial to distinguish COVID-19 from bacterial pneumonia, Mycoplasma pneumonia, and Chlamydia pneumonia in imaging diagnosis. Bacterial pneumonia mainly occupies the lung parenchyma, and here bronchial, or lobar pneumonia is the most common form. The findings of CT imaging are characterized by irregular consolidation of the pulmonary segment or subsegment, whereas ground-glass opacity is uncommon for bacterial infection [45].

\section{Treatment of COVID-19}

Glucocorticoids, antimalarial drugs, antiviral drugs, and biological agents are used in the treatment of COVID-19. Dexamethasone is a potent, long-acting, broad-spectrum glucocorticoid that acts as a synthetic version of the naturally occurring hormone cortisol [46,47]. Hydroxychloroquine and chloroquine, also known as antimalarial and anti-autoimmune agents, can also block virus infection by increasing the endosomal $\mathrm{pH}$ required for membrane fusion between virus and host cell [48]. Favipiravir, an RNA-dependent RNA polymerase inhibitor that was developed in 2002, was used in Japan in 2014 and has a strong and selective inhibitory effect against influenza viruses, which was investigated as a potential agent against RNA viruses in 2018, is also used in treatment $[49,50]$. Lopinavir-ritonavir is used as a combination of antiretroviral therapy to manage HIV-positive patients. Lopinavir inhibits HIV protease, an enzyme required for new viral assembly. Lopinavir is co-administered with ritonavir in order to prolong levels and increase exposure in the human body in consequence of its poor oral bioavailability and extensive biotransformation [48]. Remdesivir, developed against the Ebola virus in 2016, is a broad-spectrum antiviral agent that can stop viral genome replication by acting as an RNA-dependent RNA polymerase inhibitor in COVID-19 disease [48].

\section{Discussion and Conclusion}

It was an object of interest whether or not the COVID-19 outbreak, which affected the whole world, would exacerbate rheumatoid arthritis, an autoimmune disease [51]. The relationship between RA and infectious diseases is very complex. Compared with the general population, RA patients have an increased risk of infection [52]. At the beginning of the COVID-19 outbreak, concerns have arisen about the possible consequences of SARS-CoV-2 infection in patients with RA. Given the potential adverse effects of immunosuppressive drugs on viral clearance, there have been concerns that infection will be associated with increased severity of COVID-19 and excessive mortality in patients with rheumatic disease. However, the difficulty in determining the incidence of 
COVID-19 in RA patients is to review large numbers of patients and diagnose COVID-19 in terms of limitations of clinical testing [53]. We can conclude that individuals with a genetic susceptibility for rheumatoid arthritis are more likely to occur after COVID-19 infection and that the clinical course of COVID-19 may be more severe in existing RA patients than in healthy patients.

\section{Declaration of Interest}

The authors report no conflicts of interest. The authors alone are responsible for the content.

\section{References}

1. Okamoto H, Cuje TP, Yamanaka H, Kamatani N (2008) Molecular Aspects of Rheumatoid Arthritis: Role Oftranscription Factors. The Febs Journal 275(18): 4463-4470.

2. Oliver JE, Silman AJ (2006) Risk Factors for the Development of Rheumatoid Arthritis. Scand J Rheumatol 35(3): 169-174.

3. Colak A, Sertpoyraz FM, Baysol A, Girgin EM, Taylan A (2020) IschemiaModified Albumin as an Indicator of Disease Activity in Rheumatoid Arthritis. Journal of Turkish Clinical Biochemistry 18(3): 144-149.

4. Visser H (2005) Early Diagnosis of Rheumatoid Arthritis. Best Practice \& Research Clinical Rheumatology 19(1): 55-72.

5. Park SE (2020) Epidemiology, Virology, and Clinical Features of Severe Acute Respiratory Syndrome Coronavirus-2 (SARS-CoV-2; Coronavirus Disease-19). Clinical and Experimental Pediatrics 63(4): 119-124.

6. Ladner U, Pap T, Gay R, Neidhart M, Gay S (2005) Mechanisms of Disease: The Molecular and Cellular Basis of Joint Destruction in Rheumatoid Arthritis. Nature Clinical Practice 1(2): 102-110.

7. Firestein G, McInnes IB (2017) Immunopathogenesis of Rheumatoid Arthritis. Immunity 46(2): 183-192.

8. Edwards CJ (2008) Commensal Gut Bacteria and the Etiopathogenesis of Rheumatoid Arthritis. The Journal of Rheumatology 35(8): 1477-1479.

9. Sceherer HU, Häupl T, Burmester GR (2020) The Etiology of Rheumatoid Arthritis. Journal of Autoimmunity 110: 102400 .

10. Cojocaru M, Cojocaru IM, Silosi I, Vrabie CD, Tanasescu R (2010) Extraarticular Manifestations in Rheumatoid Arthritis. Maedica 5(4): 286291.

11. Kulle S (2009) The Relationship of Anti-CCP Antibody Levels with Clinical, Laboratory and Radiological Findings in Patients with Rheumatoid Arthritis. Specialization Thesis, Ankara University, Institute of Health Sciences.

12. Bes C (2015) Rheumatoid Arthritis-Associated Interstitial Lung Disease. J Turk Soc Rheumatol 7(2): 41-46.

13. Zhang M, Wang M, Tai Y, Tao J, Zhou W, et al. (2021) Triggers of Cardiovascular Diseases in Rheumatoid Arthritis. Curr Probl Cardiol 0: 100853.

14. Vreugdenhil G, Wognum AW, Eijk HG, Swaak AJ (1990) Anaemia in Rheumatoid Arthritis: The Role of Iron, Vitamin B12 and Folic Acid Deficiency and Arythropoietin Responsiveness. Annals of the Rheumatic Diseases 49(2): 93-98.

15. Keenan RT, Swearingen CJ, Yazici Y (2008) Erythrocyte Sedimentation Rate and C-Reactive Protein Levels are Poorly Correlated with Clinical Measures of Disease Activity in Rheumatoid Arthritis, Systemic Lupus Erythematosus and Osteoarthritis Patients. Clinical and Experimental Rheumatology 26(5): 814-819.
16. Pope JE, Choy EH (2021) C-Reactive Protein and Implications in Rheumatoid Arthritis and Associated Comorbidities. Seminars in Arthritis and Rheumatism 51(1): 219-229.

17. Kokubun M, Imafuku Y, Okada M, Ohguchi Y, Ashikawa T, et al. (2005) Serum Amyloid A (SAA) Concentration Varies Among Rheumatoid Arthritis Patients Estimated by SAA / CRP Ratio. Clinica Chimica Acta 360(1-2): 97-102.

18. Nakamura RM (2000) Progress in the Use of Biochemical and Biological Markers for Evaluation of Rheumatoid Arthritis. Journal of Clinical Laboratory Analysis 14(6): 305-313.

19. Ingegnoli F, Castelli R, Gualtierotti R (2013) Rheumatoid Factors: Clinical Application. Disease Markers 35(6): 727-734.

20. Block DR, Jenkins SM, Dalenberg DA, Balsanek JG, Snyder MR, et al. (2012) Analytical and Clinical Comparison of Anti-CCP Assays with Rheumatoid Factor for the Diagnosis of Rheumatoid Arthritis. Clinica Chimica Acta 413(11-12): 1015-1017.

21. Reparon Schuijt CC, Esch WJE, Kooten C, Schellekens GA, Jong B, et al. (2001) Secretion of Anti-citrulline Containing Peptide Antibody by B Lymphocytes in Rheumatoid Arthritis. Arthritis \& Rheumatism 44(1): 41-47.

22. Wang Q, Zhang Y, Wu L, Niu S, Sarkı C, et al. (2020) Structural and Functional Basis of SARS-CoV-2 Entry by Using Human ACE2. Cell 181(4): 894-904.

23. Świerkot J, Szechiński J (2006) Methotrexate in Rheumatoid Arthritis. Pharmacogical Reports 58(4): 473-492.

24. Goldenberg MM (1999) Leflunomide, A Novel Immunomodulator for the Treatment of Active Rheumatoid Arthritis. Clinical Therapeutics 21(11): $1837-1852$.

25. Dhama K, Han S, Tiwari R, Sircar S, Bhat S, et al. (2020) Coronavirus Disease 2019-COVID-19. Clinical Microbiology Reviews 33(4).

26. Schoeman D, Fielding BC (2019) Coronavirus Envelope Protein: Current Knowledge. Virology Journal 16(1): 69.

27. Duart G, García Murria MJ, Mingarro I (2021) The SARS-CoV-2 Envelope (E) Protein Has Evolved Towards Membrane Topology Robustness. BBABiomembranes 1863(7): 183608

28. Masters PS (2006) The Molecular Biology of Coronaviruses. Advances in Virus Research 66: 193-292.

29. Nile SH, Nile A, Qiu J, Li L, Jia X, et al. (2020) COVID -19: Pathogenesis, Cytokine Storm and Therapeutic Potential of Interferons. Cytokine and Growth Factor Reviews 53: 66-70.

30. Hoffmann M, Kleine Weber H, Schroeder S, Krüger N, Herrler T, et al. (2020) SARS-CoV-2 Cell Entry Depends on ACE2 and TMPRSS2 and is Blocked by a Clinically Proven Protease Inhibitor. Cell 181(2): 271-280.

31. Fehr AR, Perlman S (2015) Coronaviruses: An Overview of Their Replication and Pathogenesis. Nature Public Healty Emergency Collection 1282: 1-23.

32. Setti L, Passarini F, De Gennaro G, Barbieri P, Licen S, et al. (2020) Potential Role of Particulate Matter in the Spreading of COVID-19 in Northern Italy: First Observational Study Based on Initial Epidemic Diffusion. BMJ Open 10(9): e039338.

33. Rothan HA, Byrareddy SN (2020) The Epidemiology and Pathogenesis of Coronavirus Disease (COVID-19) Outbreak. Journal of Autoimmunity 109: 102433.

34. Jin Y, Yang H, Ji W, Wu W, Chen S, et al. (2020) Virology, Epidemiology, Pathogenesis, and Control of COVID-19. Viruses 12(4): 1-17.

35. Yang L, Liu S, Liu J, Zhang Z, Wan X, et al. (2020) COVID-19: Immunopathogenesis and Immunotherapeutics. Signal Transduction and Targeted Therapy 5(1): 128. 
36. Pechous RD, Sivaraman V, Price PA, Stasulli NM, Goldman WE (2013) Early Host Cell Targets of Yersinia Pestis During Primary Pneumonic Plaque. PLoS Pathogens 9(10): e1003679.

37. Fajgenbaum DC, June CH (2020) Cytokine Storm. The New England Journal of Medicine 383(23): 2255-2273.

38. Chen Z, Xu W, Ma W, Shi X, Li S, et al. (2021) Clinical Laboratory Evaluation of COVID-19. Clinica Chimica Acta 519: 172-182.

39. Liu DX, Liang JQ, Fung TS (2020) Human Coronavirus-229E, OC43, NL63, and HKU1 (Coronaviridae). Encyclopedia of Virology 2: 428-440.

40. Guan W, Ni Y, Liang W, Ou C, He J, et al. (2020) Clinical Characteristics of Coronavirus Disease 2019 in China. The New England Journal of Medicine 382(18): 1708-1720.

41. Yao XH, Li TY, He ZC, Ping YF, Liu HW, et al. (2020) A Pathological Report of Three COVID-19 Cases by Minimal Invasive Autopsies. Chinese Journal of Pathology 49(5): 411-417.

42. Riawati T, Indrarto W, Fauzi AR, Widitjiars W, Gunadi (2021) Various Radiological Findings in Patients with COVID-19: A Case Series. Annals of Medicine and Surgery 62: 269-273.

43. Amano Y, Kage H, Tanaka G, Gonoi W, Nakai Y, et al. (2021) Diagnostic Prediction of COVID-19 Based on Clinical and Radiological Findings in A Relatively Low COVID-19 Prevalence Area. Respiratory Investigation 59(4): 446-453.

44. Chamorro EM, Tascón AD, Sanz LI, Vèlez SO, Nacenta SB (2021) Radiologic Diagnosis of Patients with COVID-19. Radiología 63(1): 5673.

\section{ISSN: 2574-1241}

DOI: 10.26717/BJSTR.2021.38.006177

Ozan Emre EYUPOGLU. Biomed J Sci \& Tech Res

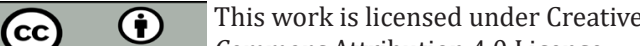

Commons Attribution 4.0 License

Submission Link: https://biomedres.us/submit-manuscript.php
45. Li J, Long X, Wang X, Fang F, Lv X, et al. (2021) Radiology Indispensable for Tracking COVID-19. Diagnostic and Interventional Imaging 102(2): $69-75$

46. Lester M, Sahin A, Pasyar A (2020) The Use of Dexamethasone in the Treatment of COVID-19. Annals of Medicine and Surgery 56: 218-219.

47. Hassan ME, Hasan HMSN, Sridharan K, Elkady A, ElSeirafi MMA (2020) Dexamethasone in Severe COVID-19 Infection: A Case Series, Respiratory Medicine Case Reports 31: 101205.

48. Lam S, Lombardi A, Ouanounou A (2020) COVID-19: A Review of the Proposed Pharmacological Treatments. European Journal of Pharmacology 886: 173451.

49. Furuta Y, Takahashi K, Fukuda Y, Kuno M, Kamiyama T, et al. (2002) In Vitro and in Vivo Activities of Anti-Influenza Virus Compound T-705. Antimicrob Agents Chemother 46(4): 977-981.

50. Delang L, Abdelnabi R, Neyts J (2018) Favipiravir As a Potential Countermeasure Against Neglected and Emerging RNA Viruses. Antiviral Research 153: 85-94

51. Terracina KA, Tan FK (2021) Flare of Rheumatoid Arthritis After COVID -19 Vaccination. The Lancet Rheumatology 3(7): e469-e470.

52. Favalli EG, Ingegnoli F, Lucia O, Cincinelli G, Cimaz R, et al. (2020) COVID-19 Infection and Rheumatoid Arthritis: Faraway, So Close!. Autoimmunity Reviews 19(5): 102523.

53. Conway R, Konig MF, Graef ER, Webb K, Yazdany J, et al. (2021) Inflammatory Arthritis in Patients with COVID-19. Translational Research 232: 49-59.

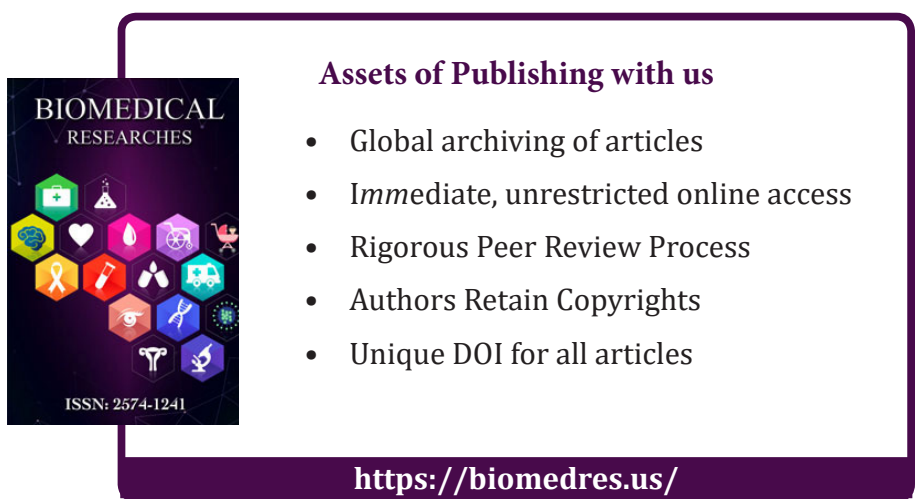

